tested cell lines. Table 1 . IC50 values of agents sorted by cell lines. N/A indicating value not achieved at threshold of $10 \mathrm{um}$. Conclusions Platinum-resistant ovarian cancer has a poor prognosis highlighting the need for new therapeutic modalities. Neither telaglenastat, savolitinib, nor AMG-232 exhibit any appreciable cytotoxicity; however, mithramycin demonstrates

\begin{tabular}{lcccc}
\multicolumn{5}{c}{ Abstract EPV001/\#119 Table 1} \\
\hline & $\begin{array}{c}\text { CAOV3 } \\
(\mathbf{n}=3)\end{array}$ & $\begin{array}{c}\text { UWB1.289 } \\
(\mathrm{n}=3)\end{array}$ & $\begin{array}{c}\text { OVCAR3 } \\
(\mathrm{n}=3)\end{array}$ & $\begin{array}{c}\text { UWB1.289-Platinum } \\
\text { Resistant(n=3) }\end{array}$ \\
\hline Cisplatin(nm) & 2733 & $2067 \pm 416$ & 4733 & $7267 \pm 2572$ \\
& \pm 208 & & \pm 1250 & \\
Mithramycin & $42.4 \pm 8$ & $57.9 \pm 46$ & $65.5 \pm 38$ & $62.2 \pm 54$ \\
$(\mathrm{~nm})$ & $\mathrm{N})$ & $\mathrm{N} / \mathrm{A}$ & $\mathrm{N} / \mathrm{A}$ & $\mathrm{N} / \mathrm{A}$ \\
Teleganestat & $\mathrm{N} / \mathrm{A}$ & $\mathrm{N} / \mathrm{A}$ & $\mathrm{N} / \mathrm{A}$ & $\mathrm{N} / \mathrm{A}$ \\
Savolitinib & $\mathrm{N} / \mathrm{A}$ & $\mathrm{N} / \mathrm{A}$ & $\mathrm{N} / \mathrm{A}$ & $\mathrm{N} / \mathrm{A}$ \\
AMG-232 & $\mathrm{N} / \mathrm{A}$ & & & \\
\hline
\end{tabular}

cytotoxicity in the low nanomolar range in several representative ovarian cancer cell lines, including two platinum-resistant lines. The potential therapeutic benefit of mithramycin warrants further preclinical evaluation.

\section{EPV002/\#308 METABOLOMIC ADAPTATIONS ASSOCIATED WITH CHEMORESISTANCE IN OVARIAN CANCER CELL LINES}

${ }^{1} \mathrm{~L}$ Corey ${ }^{*},{ }^{2} \mathrm{G}$ Mor, ${ }^{1} \mathrm{~L}$ Matherly, ${ }^{3} \mathrm{~J}$ Jiang, ${ }^{3} \mathrm{Y} Y$ Yue, ${ }^{4} \mathrm{~N}$ Tiwari, ${ }^{1} \mathrm{Z}$ Hou, ${ }^{1} \mathrm{Y}$ You, ${ }^{3} \mathrm{~J} \mathrm{Li},{ }^{5} \mathrm{~S}$ Kim, ${ }^{1} \mathrm{R}$ Rattan, ${ }^{1} \mathrm{~A}$ Alvero, ${ }^{6} \mathrm{R}$ Gogoi. ${ }^{1}$ Wayne State University, Oncology, Detroit, USA; ${ }^{2}$ Wayne State University, Obstetrics and Gynecology, Detroit, USA; ${ }^{3}$ Wayne State University, Metabolomics, Detroit, USA; ${ }^{4}$ Henry Ford Health Systems, Oncology, Detroit, USA; ${ }^{5}$ Wayne State University, Biostatistics, Detroit, USA; ${ }^{6}$ Wayne State University/Karmanos Cancer Institute. Division of Gynecologic Oncology, Gyn Oncology, Northville, USA

\subsection{6/ijgc-2021-IGCS.69}

Objectives Over $80 \%$ of ovarian cancer (OC) patients will experience relapse after an initial response to platinum-based chemotherapy. Acquisition of metabolomic adaptations is
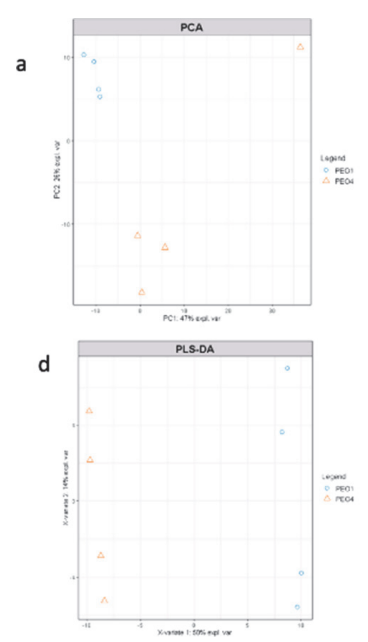
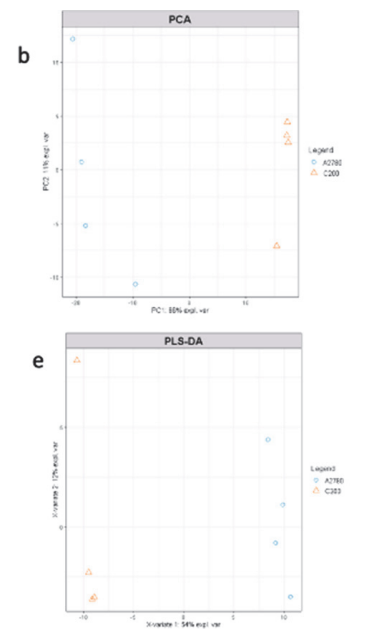
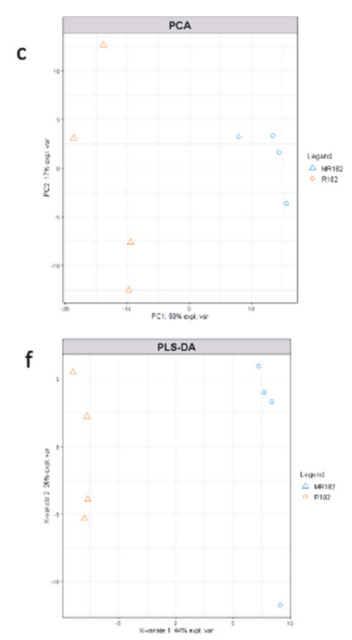

Abstract EPV002/\#308 Figure 1 PCA of a. PE01 vs PE04, b. A2780 vs C200, c. MR182 vs R182; PLS-DA of d. PE01 vs PE04, e. A2780 vs C200, f. MR182 vs R182
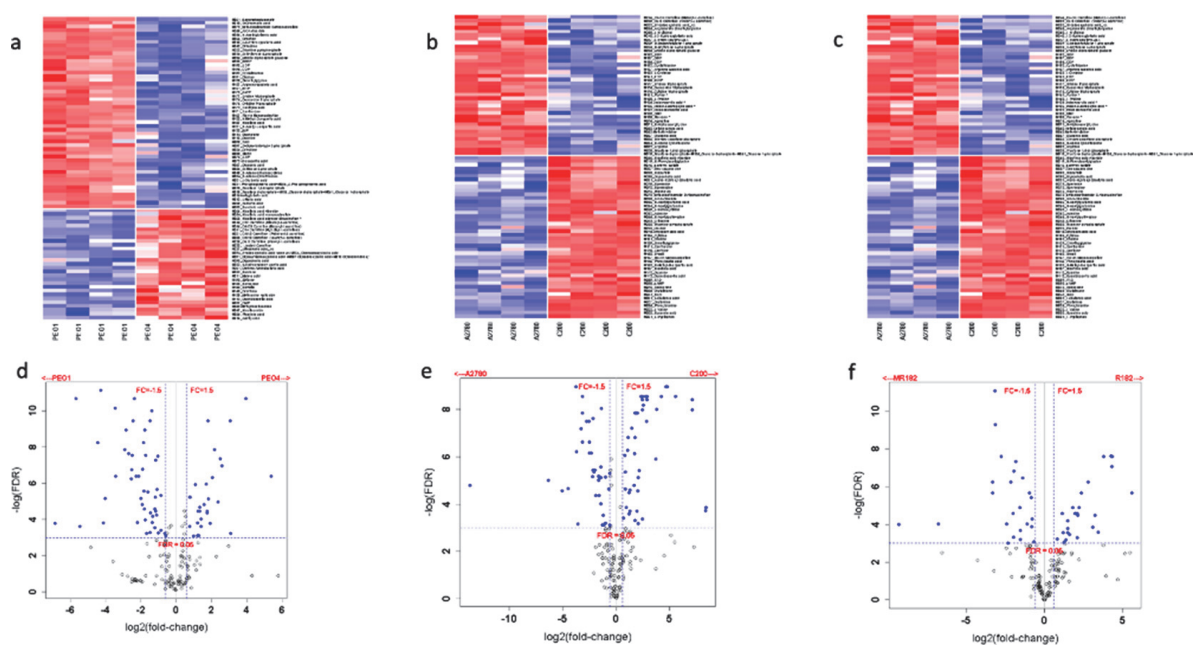

Abstract EPV002/\#308 Figure 2 Heat map of a. PE01 vs PEO4, b. A2780 vs C200, c. MR182 vs R182; Volcano plot with FDR $\leq 5 \%$ and absoulute fold-change $\geq 1.5$ of $d$. PE01 vs PE04, e. A2780 vs C200, f. MR182 vs R182 
thought to be an integral part of chemoresistance, but the relation of these adaptations to chemoresistance is poorly understood. Our aim was to identify the metabolic adaptations that are specifically associated with platinum-resistant (PR) cell lines and its platinum-sensitive (PS) derivatives across multiple OC cell lines.

Methods Targeted metabolic analysis evaluating 242 metabolites of the PS A2780, PEO1, and mR182 cell lines was performed along with their respective PR derivatives, C200, PEO4, R182. The group comparison was performed using unpaired t-tests followed by FDR correction. The differentially expressed metabolites were identified using two criteria: FDR $\leq 5 \%$ and absolute fold-change $\geq 1.5$. The pathway analysis was performed using Metaboanalyst ${ }^{\mathrm{TM}}$ with the metabolites that have unadjusted $\mathrm{p}$-value $\leq 5 \%$.

Results Many significantly impacted pathways were conserved among the PR cell lines. Compared to the PS counterparts, the PR PEO4, C200, and R182 lines had metabolite concentrations with $\mathrm{FC} \geq 1.5$ in 29,44 , and 28 measured metabolites, respectively. The top pathways impacted were 'nicotinate and nicotinamide metabolism', 'purine metabolism', and 'phenylalanine, tyrosine, tryptophan biosynthesis'. A global analysis of PS vs PR was performed. The top five significantly impacted pathways were: Arginine biosynthesis, Pyrimidine and Purine metabolism, Phenylalanine, tyrosine and tryptophan biosynthesis' and 'Starch and sucrose metabolism'.
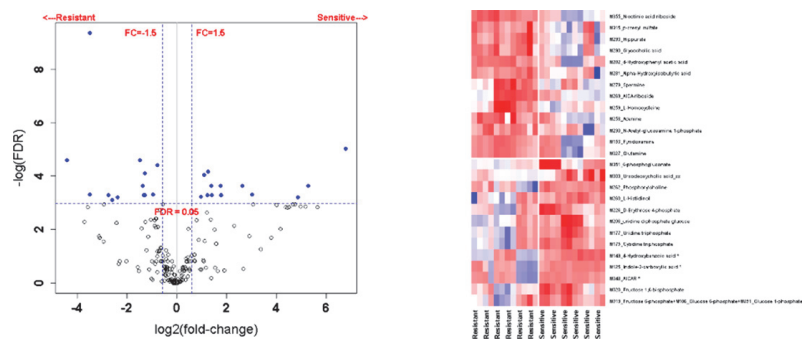

Abstract EPV002/\#308 Figure 3 a) Volcano plot of sensitive cohort vs resistant cohort; b) Heat map of sensitive cohort vs resistant cohort

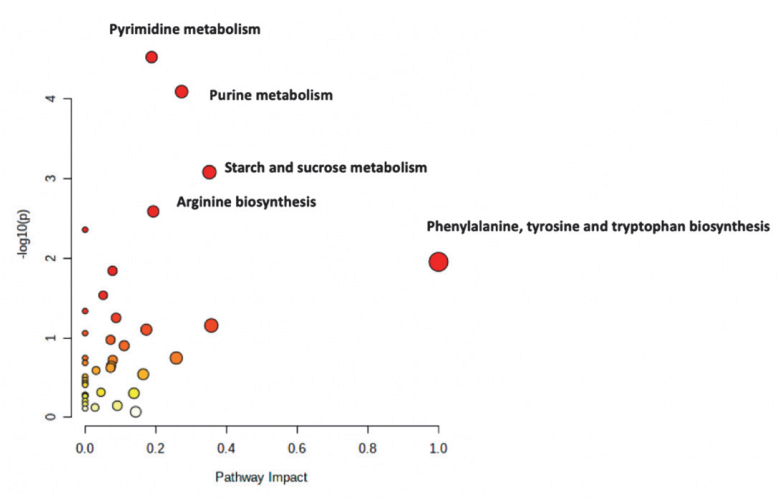

Abstract EPV002/\#308 Figure 4 Top impacted pathways of combined PR cohort compared to PS cohort

Conclusions We identified multiple shared metabolomic pathways among established PR OC cell lines that highlight conserved motifs of PR. These may represent targetable pathways to predict or reverse chemoresistance.

\section{EPV003/\#326 AN INTEGRATED GENOMIC, PROTEOMIC AND IMMUNOPEPTIDOMIC APPROACH TO DISCOVER NOVEL TUMOUR NEOANTIGENS IN AN IMMUNOLOGICALLY COLD OVARIAN CANCER FOR PERSONALISED T-CELL RECEPTOR THERAPY}

${ }^{1,2} \mathrm{GY} \mathrm{Ho}{ }^{*},{ }^{3} \mathrm{P}$ Faridi, ${ }^{1} \mathrm{~J} \mathrm{Wu},{ }^{4,5} \mathrm{H}$ Barker, ${ }^{1,6} \mathrm{~T}$ Nguyen-Dumont, ${ }^{1} \mathrm{~J}$ Chang, ${ }^{1} \mathrm{~A}$ Fell, ${ }^{1} \mathrm{P}$ Eggenhuizen, ${ }^{1} \mathrm{~J}$ Steen, ${ }^{2} \mathrm{~T}$ Manolitsas, ${ }^{1,2} \mathrm{~S}$ Frentzas, ${ }^{4,7} \mathrm{~J}$ Bedo, ${ }^{4,5} \mathrm{C}$ Vandenberg, ${ }^{4,5} \mathrm{~T}$ Papenfuss, ${ }^{4,5} \mathrm{C}$ Scott, ${ }^{1} \mathrm{~J}$ Ooi, ${ }^{1,2}$ E Segelov. 'Monash University, School of Clinical Sciences, Clayton, Australia; ${ }^{2}$ Monash Health, Oncology Department, Clayton, Australia; ${ }^{3}$ Monash University, Monash Biomedicine Discovery Institute, Clayton, Australia; ${ }^{4}$ Walter and Eliza Hall Institute of Medical Research, Cancer Biology and Stem Cells Division, Parkville, Australia; ${ }^{5}$ University of Melbourne, Department of Medical Biology, Parkville, Australia; ${ }^{6}$ University of Melbourne, 6. Departcomputing and Information Systems, Parkville, Australia; 'University of Melbourne, Department of Computing and Information Systems, Parkville, Australia

\subsection{6/ijgc-2021-IGCS.70}

Objectives Ovarian carcinosarcoma (OCS) are rare aggressive cancers with poor prognosis and limited effective treatments. The tumour mutation burden in OCS is often low. Therefore, these tumours are immunologically 'cold' and relatively irresponsive to single agent immunotherapy. We explored tumour neoantigen discovery in an OCS using various genomic and proteomic platforms for personalised T-cell receptor (TCR) therapy.

Methods Whole genome sequencing (WGS) was performed on SFRC01177 OCS tumour specimen taken at surgery. Fresh tumour specimens obtained at surgery and biopsy at recurrence were engrafted subcutaneously in NOD-scidIL2Rgammanull (NSG) to generate a paired patient derived xenograft (PDX) model. Whole exome sequencing and RNA sequencing (WES/RNAseq) together with nano-ultra-performance liquid chromatography coupled to high-resolution mass spectrometry were performed on the snap frozen tumours from the baseline and recurrent PDX for tumour neoantigen (TNA) discovery.

Results A total of 6,500 mutant TNA were predicted in silico from the baseline WGS data which were narrowed down to 65 and 33 respectively based on the baseline and recurrent PDX tumours WES/RNAseq data. The immunopeptidomic analysis revealed over 100 major histocompatibility complex bound antigens including mutant, spliced and cancer testis antigens. The PDX was re-established in NSG MHC ${ }^{\text {null }}$ mouse model and was shown to retain the platinum refractory in vivo response as well as to tolerate 1 million HLA-matched donor CD8 + T-cell injections.

Conclusions We have discovered multiple tumour specific neoantigens using the comprehensive TNA discovery platforms, which will direct our TCR engineering. In parallel, we have also established an OCS PDX model suitable for cell-based therapy testing.

\section{EPV004/\#360 INHIBITION OF CANCER CELL-DEPENDENT GLYCOLYSIS THROUGH AVB-500, A SELECTIVE INHIBITOR OF GAS6-AXL, IN COMBINATION WITH PACLITAXEL IN HIGH-GRADE ENDOMETRIAL CANCER}

${ }^{1} S$ Bruce*, ${ }^{1} \mathrm{E}$ Lomonosova, ${ }^{1} \mathrm{H}$ Noia, ${ }^{1} \mathrm{E}$ Stock, ${ }^{2} \mathrm{~K}$ Cho, ${ }^{1} \mathrm{D}$ Khabele, ${ }^{1} \mathrm{~L}$ Kuroki, ${ }^{1} \mathrm{~A}$ Hagemann, ${ }^{1} \mathrm{C}$ Mccourt, ${ }^{1} \mathrm{P}$ Thaker, ${ }^{1} \mathrm{D}$ Mutch, ${ }^{1} \mathrm{M}$ Powell, ${ }^{2} \mathrm{~L}$ Shriver, ${ }^{2} \mathrm{G}$ Patti, ${ }^{1} \mathrm{~K}$ Fuh. 'Washington University, Gynecologic Oncology, St. Louis, USA; ${ }^{2}$ Washington University, Chemistry, St. Louis, USA

10.1136/ijgc-2021-IGCS.71 